\title{
Tumour type and size are high risk factors for the syndrome of "cerebellar" mutism and subsequent dysarthria
} Coriene E Catsman-Berrevoets, Hugo R Van Dongen, Paul G H Mulder,
Daniel Paz y Geuze, Philippe F Paquier, Maarten H Lequin
Department of Child Neurology C E Catsman-Berrevoets H R Van Dongen

Department of Child Neurosurgery D Paz y Geuze

Department of Child Radiology, Sophia Children Hospital, University Hospital Rotterdam, The

Netherlands M H Lequin

Department of Biostatistics and Epidemiology, Erasmus University Rotterdam, The Netherlands P G H Mulder

Department of Neurology University Hospital Erasme, Brussels, Belgium, and Department of Ear Nose and Throat Surgery, University of Antwerp, School of Medicine, Antwerp, Belgium

P F Paquier

Correspondence to:

Dr C E Catsman-Berrevoets, Department of Child Neurology, University Hospital Rotterdam, Sophia Children's Hospital, PO Box 2060, 3000 CB Rotterdam, The Netherlands. Telephone 0031104636342 ; fax 0031

10 4636345; email

Catsman@neur.azr.nl

Received 29 October 1998 and in final form

18 June 1999

Accepted 29 June 1999

\begin{abstract}
Objective- "Cerebellar mutis" and subsequent dysarthria (MSD) is a documented complication of posterior fossa surgery in children. In this prospective study the following risk factors for MSD were assessed: type, size and site of the tumour; hydrocephalus at presentation and after surgery, cerebellar incision site, postoperative infection, and cerebellar swelling. Methods-In a consecutive series of 42 children with a cerebellar tumour, speech and neuroradiological studies (CT and MRI) were systematically analysed preoperatively and postoperatively. Speech was assessed using the Mayo Clinic lists and the severity of dysarthria using the Michigan rating scale.

Results-Twelve children (29\%) developed MSD postoperatively. The type of tumour, midline localisation, and vermal incision were significant single independent risk factors. In addition, an interdependency of possible risk factors (tumour $>5 \mathrm{~cm}$, medulloblastoma) was found.

Conclusion-MSD often occurs after paediatric cerebellar tumour removal and is most likely after removal of a medulloblastoma with a maximum lesion diameter $>5 \mathrm{~cm}$.

(f Neurol Neurosurg Psychiatry 1999;67:755-757)
\end{abstract}

Keywords: mutism and subsequent dysarthria; cerebellar tumour; risk factors

Acquired mutism, defined as a condition of complete absence of speech that is not associated with other aphasic symptomatology or alteration of consciousness ${ }^{1}$ may occur in various neurological conditions with different aetiologies. ${ }^{2}$ Neurosurgical interventions such as callosotomy, resection of the supplementary motor cortex of the dominant hemisphere, and cerebellar mass lesion resection are also associated with postoperative mutism. ${ }^{1}$

In a previous study we assessed the speech of 15 children immediately after removal of a cerebellar tumour. ${ }^{3}$ Five of them developed the syndrome of "cerebellar" mutism and subsequent dysarthria (MSD). The core features of this predominantly paediatric syndrome are: (1) it occurs after resection of a cerebellar mass lesion; (2) there is delayed onset of mutism after a brief interval of 1-2 days of relatively normal speech postsurgery; (3) there is transient mutism that lasts from 1 day to 6 months, followed by a severe dysarthria, which recovers completely in 1-3 months; (4) frequent association with other neurological manifestations, such as long tract signs and neurobehavioural abnormalities. $^{34}$

The mechanisms for the development of MSD are still unknown. In retrospective studies various risk factors have been suggested such as tumour size, length of the vermian incision at surgery, and postoperative complications such as oedema within the pontine tegmentum or brachium pontis, ${ }^{4}$ hydrocephalus, and meningitis. ${ }^{5-7}$

In this prospective study we examined whether type, size, and site of the tumour, site of the cerebellar incision, preoperative and postoperative hydrocephalus, postoperative infection, and oedema are related to MSD. In addition we assessed the effects of sex and age for MSD.

\section{Patients and methods}

PAEDIATRIC POPULATION

Forty two children (29 boys and 13 girls, age range 2-17 years) with a normal psychomotor development and age appropriate speech, were admitted for a first resection of a cerebellar tumour. In 17 children a diagnosis of medulloblastoma was made. In 21 children the tumour was found to be an astrocytoma, in three an ependymoma and in one a haemangioblastoma. In line with the literature no speech problems had been found by the parents before admission. ${ }^{4-10}$

\section{DATA EVALUATION}

In all children, speech behaviour (in very young children elicited by, and in response to, their parents) was followed up every day during the first 2 weeks after surgery - that is, until the risk of developing MSD became almost zero. Speech behaviour was recorded on videotape before and immediately after surgery, at 3 days after surgery, and, in cases of mutism at as short as possible intervals for 4 weeks. Subsequently these patients were assessed every 2 nd week until the recurrence of speech.

Speech (spontaneous language, repetition of words and sentences, and-if possiblereading aloud) was assessed according to the "Mayo Clinic lists" of Darley et al, in which speech characteristics such as voice quality, nasality, articulation, speech rate, and respiration are included. ${ }^{11}$ For rating the severity of the dysarthric components from videotaped samples of spontaneous speech, we used the 
Table 1 Results of analysis of single risk factors

\begin{tabular}{|c|c|c|c|}
\hline Risk factor & MSD present & MSD absent & $p$ Value \\
\hline \multicolumn{4}{|l|}{ Tumour type: } \\
\hline Medulloblastoma & 9 & 8 & \multirow[t]{2}{*}{0.006} \\
\hline Other tumour type & 3 & 22 & \\
\hline \multicolumn{4}{|l|}{ Tumour site: } \\
\hline Involving cerebellar midline & 12 & 16 & \multirow[t]{2}{*}{0.003} \\
\hline Not involving cerebellar midline (lateral) & 0 & 14 & \\
\hline \multicolumn{4}{|l|}{ Incision site: } \\
\hline Vermal incision & 10 & 12 & \multirow[t]{2}{*}{0.028} \\
\hline Lateral to vermis & 2 & 18 & \\
\hline \multicolumn{4}{|l|}{ Postoperative infection: } \\
\hline Present & 2 & 3 & \multirow[t]{2}{*}{0.613} \\
\hline Absent & 10 & 27 & \\
\hline Size $(\mathrm{cm})^{\star}$ & $5.4(0.87)$ & $5.4(1.32)$ & 0.789 \\
\hline BI before surgery* & $0.267(0.065)$ & $0.238(0.079)$ & 0.198 \\
\hline BI after surgery ${ }^{\star}$ & $0.150(0.61)$ & $0.162(0.074)$ & 0.562 \\
\hline Mean age $(y)^{\star}$ & $7.3(4.4)$ & $7.8(4.12)$ & 0.764 \\
\hline \multicolumn{4}{|l|}{ Sex: } \\
\hline Boys & 8 & 21 & \multirow[t]{2}{*}{1.0} \\
\hline Girls & 4 & 9 & \\
\hline
\end{tabular}

University of Michigan rating system. ${ }^{12}$ Neurological examination of the speech musculature was carried out according to the protocol for studying acquired childhood dysarthria. ${ }^{13-16}$

Brain CT, or MRI, or both were studied to determine the preoperative maximum lesion diameter ${ }^{4}$ and site of the tumour. We computed the bicaudate index (BI) as a measure of ventricle dilatation at presentation and within 1 week after surgery. ${ }^{17}$ Data on the occurrence of postoperative meningitis and oedema of the brain stem were also collected.

\section{STATISTICAL ANALYSIS}

In a preliminary analysis the relation of each variable to MSD was separately tested by Fisher's exact test (or $\chi^{2}$ test) for categorical nominal variables and the Mann-Whitney $U$ test for ordinally scaled variables, using the SPSS/PC+ statistical package.

The combined effect of the single variables on MSD, which in the preliminary analysis seemed to be relevant, was analysed by exact logistic regression analyses. For this we used the software package LogXact of CYTEL Software Corporation, based on an algorithm developed by Hirji et al. ${ }^{18}$ For statistical significance mid-p values were used in the exact analyses. Because of the relatively small series of patients an exact analysis is indicated with dichotomisation of some multilevel categorical variables. The variables tumour type and site and incision site were dichotomised as follows: tumour type was divided into medulloblastoma versus other tumour types. Tumour site was defined as tumour localisation in the cerebellar midline with or without tumour extension into the cerebellar hemisphere, versus tumour localisation strictly confined to the cerebellar hemisphere - that is, lateral. Incision site was subdivided into vermal incision versus incision laterally to the vermis.

\section{Results}

The MSD syndrome occurred in 12 (29\%) out of 42 children. It occurred in nine of 17 (53\%) children (four girls, five boys) with a medulloblastoma, one of three boys (33\%) with an ependymoma, and two boys out of $21(11 \%)$ children with a pilocytic astrocytoma.
Table 2 Results of exact logistic regression analysis in terms of $p$ values

\begin{tabular}{ll}
\hline & $p$ Value \\
\hline Tumour site cerebellar midline & 0.04 \\
Tumour size $(\mathrm{cm})$ & 0.26 \\
Interaction medulloblastoma×size & 0.05 \\
\hline
\end{tabular}

Table 3 Interaction of size and type of tumour

\begin{tabular}{lll}
\hline & MSD present & MSD absent \\
\hline Medulloblastoma: & 2 & 7 \\
Size $<5 \mathrm{~cm}$ & 7 & 1 \\
$>5 \mathrm{~cm}$ & & \\
Other tumour type: & 2 & 10 \\
Size $<5 \mathrm{~cm}$ & 1 & 12 \\
$>5 \mathrm{~cm}$ & \\
\hline
\end{tabular}

Sizes of medulloblastomas ranged from 3.2$7.2 \mathrm{~cm}$ (mean $4.96 \mathrm{~cm}$ ). Sizes of the tumours in the other tumour type group ranged from 4.0$8.0 \mathrm{~cm}$ (mean $5.64 \mathrm{~cm}$ ).

The duration of the mutism varied from 1 day to 5 months.

For each single variable assessed separately, table 1 gives the statistical results according to the presence or absence of MSD. In addition table 1 shows that sex and age were not related to MSD. In many cases artefacts on postoperative CT obscured the exact delineation of oedema in the brainstem. Therefore these data could not be carefully assessed and evaluated as single risk factors.

Because of possible interdependencies between risk factors, the simultaneous effect of the variables listed in table 1 was subsequently analysed by exact logistic regression analyses. These analyses showed that tumour size, although not a significant risk factor for MSD when considered separately, reached significance exclusively in combination with medulloblastoma (table 2). Hence this suggests an interaction between size and medulloblastoma. The other explanatory variables did not give a significant contribution when additionally included in the model.

In the logistic regression model ${ }^{18}$ calculations showed that if the tumour site was cerebellar midline the odds of developing MSD was multiplied by $8.2 \quad(p=0.004)$ versus tumour localisation laterally in the cerebellar hemisphere. In the medulloblastoma group each increase of tumour size by $1 \mathrm{~cm}$ meant a multiplication of the odds to develop MSD by $1.76(\mathrm{p}=0.05)$, given site.

In table 3 the interaction found in the logistic regression analysis between size and type as risk factors for MSD is empirically demonstrated on the basis of the raw data. In this table size is dichotomised as lower or higher than the median $(5 \mathrm{~cm})$. From these data, it may be concluded that size only has an effect in the medulloblastoma group, or in other words: medulloblastoma is a risk factor for MSD only when the size is large.

\section{Discussion}

Until now risk factors of MSD syndrome were exclusively considered separately. ${ }^{45}{ }^{19-22}$ Our study clearly shows that a complex mechanism 
is responsible for MSD, as simultaneous analyses of several factors disclose the importance of their interactions.

By contrast with the manifest interaction of tumour type and size in the medulloblastoma group, such an interaction was not found in the other tumour type group. Because MSD after resection of astrocytoma or ependymoma also occurs, other single risk factors or even more complex interactions of two or more risk factors may be presumed. Pollack suggests that MSD after a midline surgical approach to the tumour is caused by excessive paravermal manipulation of tissue and subsequent bilateral damage to the dentate and interpositus nuclei, rather than by damage to the vermis. ${ }^{6}$ This assumption is based on the study of Fraioli and Guidetti who reported mutism after bilateral stereotaxic lesions in the region of the dentate and interpositus nuclei. ${ }^{23}$ Their findings suggest that the intactness of at least one set of deep cerebellar nuclei is necessary to avoid MSD. This would explain the absence of MSD in cases of even very large pilocytic astrocytomas situated laterally in the cerebellar hemisphere. In this context it is of interest to note that Kellogg and Piatt recently described an alternative method for resection of fourth ventricle tumours by avoiding a midvermal approach. ${ }^{24}$ They claimed that this cerebellomedullary fissure approach minimised the risk of neurological complications, including MSD. In a previous smaller study of ours preoperative hydrocephalus (hydrocephalus clinically needing aventriculoperitoneal shunt) was associated with MSD. ${ }^{3}$ This finding is not confirmed in the present study.

Obviously other risk factors, which as yet have not been identified, are crucial for the development of MSD. One factor might be delayed transient ischaemia of the median cerebellar structures as in one patient SPECT showed hypoperfusion of supratentorial frontotemporal cortical regions. ${ }^{16}$ This finding suggests that postoperative diaschisis plays an important part in the development of MSD, probably by way of the dentatothalamocortical circuitry.

We found an even higher incidence of MSD $(29 \%)$, than the $8 \%-13 \%$ reported in the literature. ${ }^{457}$ This may be explained by the prospective nature of our study. We assessed all children every day immediately after surgery for the first 2 weeks, so that the risk of missing children with rather brief periods of mutism was kept minimal. The higher incidence of MSD in our series cannot be attributed to an overrepresentation of medulloblastoma, as the distribution of tumour types is in agreement with epidemiological data. ${ }^{25}$

Conclusions

Acquired mutism in childhood is a complex phenomenon occurring in various neurological conditions with different aetiologies. The MSD syndrome has recently been described in many case studies. Our study shows the complex interaction of tumour type and size: MSD occurs most often after removal of a medulloblastoma with a lesion diameter $>5 \mathrm{~cm}$.

We are indebted to Viviane de Martelaere for her valuable advice with respect to the presentation of the statistical findings.

1 Crutchfield JS, Sawaya RS, Meyers CA, et al. Postoperative mutism in neurosurgery. $\mathcal{F}$ Neurosurg $1994 ; 81: 115-21$.

2 Van Mourik M, Van Dongen HR, Catsman-Berrevoets CE. The many faces of acquired neurological mutism in childhood. Pediatr Neurol 1996;15:352-7.

3 Van Dongen HR, Catsman-Berrevoets CE, Van Mourik M. The syndrome of "cerebellar" mutism and subsequent dysarthria. Neurology 1994;44:2040-6.

4 Pollack IF, Polinko P, Albright AL, et al. Mutism and pseudobulbar symptoms after resection of posterior fossa tumors in children: incidence and pathophysiology. Neurosurgery 1995;37:885-93.

5 Dailey AT, McKhann GM, Berger MS. The pathophysiology of oral apraxia and mutism following posterior fossa tumor resection in children. $\mathcal{F}$ Neurosurg 1995;83:467-75.

6 Pollack IF. Posterior fossa syndrome. In: Schmahmann JD, ed. The cerebellum and cognition: international review of neurobiology. San Diego: Acadamic Press, 1997:41:411-32.

7 Van Calenbergh F, Van de Laar A, Plets C, et al. Transient cerebellar mutism after posterior fossa surgery in children. Neurosurgery 1995;37:894-8.

8 Halperin EC, Friedman HS. Is there a correlation between duration of presenting symptoms and stage of medulloblas toma at the time of diagnosis. Cancer 1996;78:874-80.

9 Kulkarni AV, Becker LE, Jay V, et al. Primary cerebellar glioblastomas multiforme in children. F Neurosurg 1999;90: 546-50.

10 Pencalet P, Maixner W, Sainte-Rose C, et al. Benign cerebellar astrocytomas in children. F Neurosurg 1999;90: 265-73.

11 Darley FL, Aronson AE, Brown JK. Differential diagnostic patterns of dysarthria. $\mathcal{F}$ Speech Hearing Res 1969;12:24669.

12 Kluin KJ,Gilman S, Markel DS, et al. Speech disorders in olivoponto-cerebellar atrophy correlate with positron emission tomography findings. Ann Neurol 1988;23:285-90.

13 Bak E, Van Dongen HR, Arts WFM. The analysis of acquired dysarthria in childhood. Dev Med Child Neurol 1983;25:81-7.

14 Van Dongen HR, Arts WFM, Youssef-Bak E. Acquired dysarthria in childhood. An analysis of dysarthric features in relation to neurologic deficits. Neurology 1987;37:296-9.

15 Catsman-Berrevoets CE, Van Dongen HR, Zwetsloot CP.Transient loss of speech followed by dysarthria after removal of posterior fossa tumour. Dev Med Child Neurol 1992;34:1102-7.

16 Van Mourik M, Catsman-Berrevoets CE, Van Dongen HR, et al. Complex orofacial movements and the disappearance of cerebellar mutism. Report of five cases. Dev Med Child Neurol 1997;39:686-90.

17 Van Gijn J, Hijdra A, Wijdicks EFM, et al. Acute hydrocephalus after aneurysmal subarachnoid hemorrhage. f Neurosurg 1985;63:355-62.

18 Hirji KF, Mehta CR, Patel NR. Computing distributions for exact logistic regression. Fournal of the American Statistical Association 1987;82:1110-7.

19 Aguiar PH, Plese JPP, Ciquini O, et al. Transient mutism following posterior fossa approach to cerebellar tumors in children: a critical review of the literature. Childs Nerv Syst 1995;11:306-10.

20 Ersahin Y, Mutluer S, Saydam S, et al. Cerebellar mutism: report of two unusual cases and review of the literature. Clin Neurol Neursurg 1997;99:130-4.

21 Ferrante L, Mastronardi L, Acqui M, et al. Mutism after posterior fossa surgery. F Neurosurg 1990;72:959-63.

22 Rekate HL, Grubb RL, Aram DM, et al. Muteness of cerebellar origin. Arch Neurol 1985;42:697-8.

23 Fraioli A, Guidetti B. Effects of stereotactic lesions of the dentate nucleus of the cerebellum in man. Appl Neurophysiol 1975;38:81-90.

24 Kellogg JX, Piatt JH. Resecton of fourth ventricle tumors without splitting the vermis: the cerebellomedullary fissure approach. Pediatr Neurosurg 1997;27:28-33.

25 Koos WT, Miller MH. Intracranial tumors of infants and children. St Louis: Mosby, 1971. 